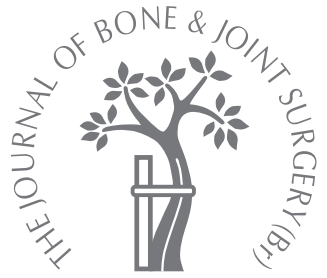
S. Roy,
P. Dobson,
L. Henry

From the Wakefield Orthopaedic Clinic and Gribbles

Pathology, Adelaide, South Australia
S. Roy, MBChB, MPhil, FRCS(Tr \& Orth), Fellow in Lower Limb and Sports Surgery

P. Dobson, FRACS FAOrthA, Consultant Orthopaedic Surgeon

Wakefield Orthopaedic Clinic, 270 Wakefield Street Adelaide 5000, South Australia.

L. Henry, FRCPA,

Consultant Histopathologist Gribbles Pathology, 1

Goodwood Road, Wayville, Adelaide 5034, South Australia.

Correspondence should be sent to Mr S. Roy; e-mail: wsturoy:aol.com

(C)2005 British Editorial Society of Bone and Joint Surgery

doi:10.1302/0301-620X.87B10. $16493 \$ 2.00$

$J$ Bone Joint Surg [Br] 2005:87-B:1423-4.

Received 9 March 2005

Accepted 28 April 2005

\title{
An isolated osteochondroma with underlying non-Hodgkin's lymphoma of bone
}

\begin{abstract}
Osteochondroma is the most common benign bone tumour. The risk of sarcomatous change in an isolated lesion is approximately $1 \%$. We report a case of an isolated osteochondroma which appeared benign on clinical and plain radiographic examination but routine histological analysis revealed non-Hodgkin's lymphoma in the underlying bone. This association has not previously been reported and the case emphasises the importance of routine histological analysis, even if a lesion appears benign.
\end{abstract}

Osteochondroma represents a developmental dysplasia of a growth plate in the form of a cartilage capped bony projection from the surface of the affected bone. It is more common in long bones but may occur in any bone formed by enchondral ossification. The risk of sarcomatous change in an isolated lesion is approximately $1 \%{ }^{1}$

A review of the literature revealed one case of high-grade non-Hodgkin's lymphoma within an exostosis in a patient with multiple hereditary exostoses ${ }^{2}$ but to our knowledge, the association of non-Hodgkin's lymphoma and an isolated exostosis has not been previously described.

\section{Case report}

A 20-year-old university student presented with a long history of a lump on the medial aspect of his knee. It had not caused any symptoms until the year prior to presentation. A diagnosis of a solitary exostosis had been made five years previously. In the year prior to his presentation, he had experienced increasing symptoms of pain around the lump especially after any sporting activity.

Clinical examination revealed a tender lump deep to vastus medialis just above the right knee. It was not tender. There was no associated effusion and he had a full range of movement of the knee. General examination showed

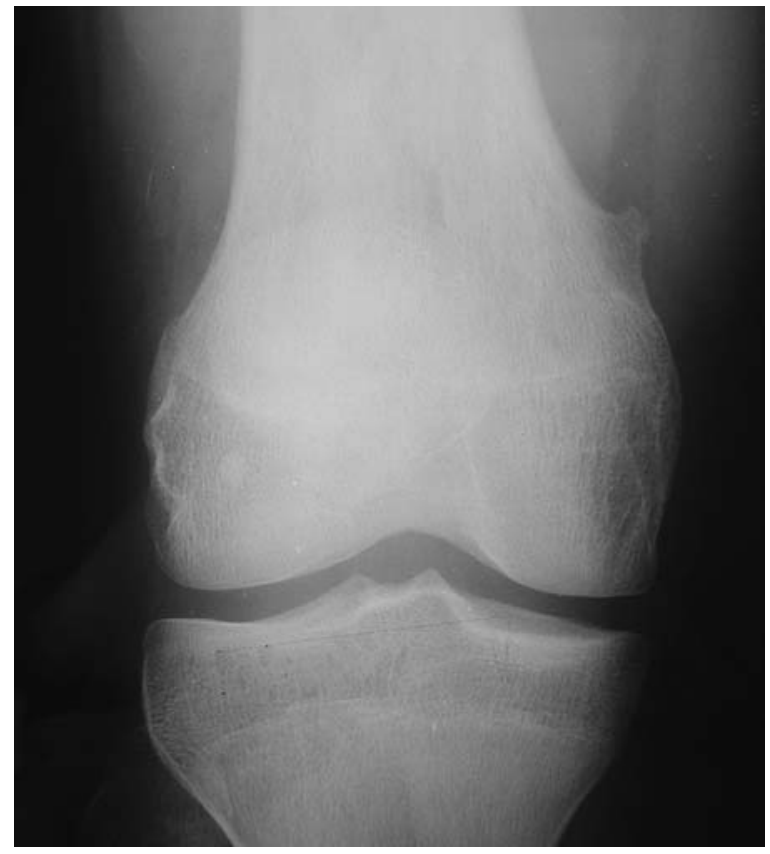

Fig. 1
An anteroposterior radiograph of the knee showing an isolated osteochondroma. 


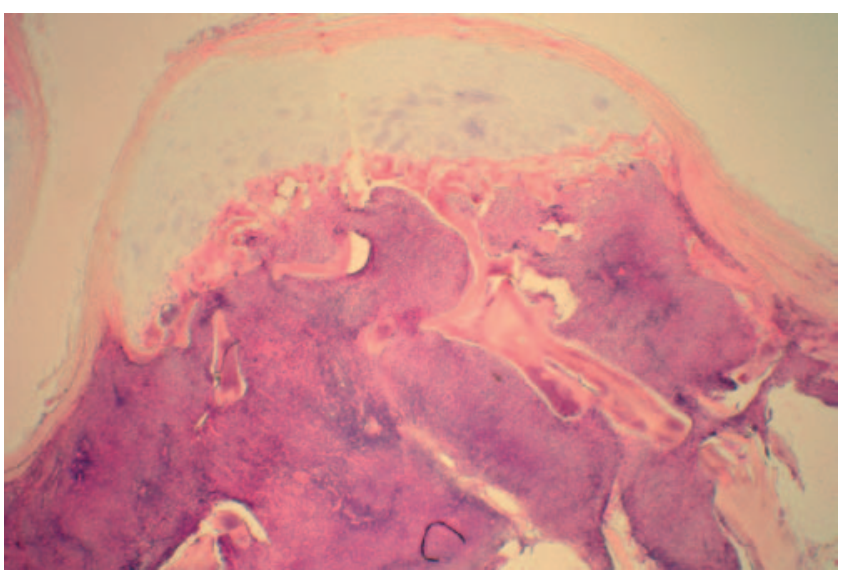

Fig. 2

Histological slide of lesion showing cartilage cap and underlying tumour (x2).

this to be an isolated lesion. Plain radiographs confirmed the presence of a pedunculated osteochondroma arising from the distal medial aspect of the right femur (Fig. 1).

The patient requested excision of the lesion and he underwent surgery three months later. Under general anaesthesia with the leg exsanguinated, the lesion was approached from the medial aspect. The vastus medialis was reflected from the lesion. The lesion looked benign, was excised with an osteotome and sent for histological analysis. The patient made an uneventful recovery and was allowed home the next day.
To our surprise, routine histological analysis revealed a large cell, non-Hodgkin's lymphoma within the bony portion of the lesion (Fig. 2). An MRI and bone scan showed that the lymphoma was isolated to the distal femur which was confirmed with further staging. To date he has had four cycles of cytotoxic chemotherapy and is due to have consolidation radiotherapy in the near future.

\section{Discussion}

The risk of sarcomatous change within an isolated osteochondroma is very small. ${ }^{1}$ Isolated osteochondromata may be left alone or excised if they cause symptoms due to irritation of muscle, tendon or nerve. Even though the clinical and radiographic appearances of the lesion in our case were benign, routine histological analysis revealed a large cell, non-Hodgkin's lymphoma. Appropriate investigation, staging and treatment were begun. To our knowledge the association of non-Hodgkin's lymphoma and solitary exostosis has not been previously reported.

The great rarity of this coincidence does not raise the prospect of pathological association; but the case emphasises the importance of histological analysis even if the lesion in question appears benign.

No benefits in any form have been received or will be received from a commercial party related directly or indirectly to the subject of this article.

\section{References}

1. Guidici MA, Moser RP Jr, Kransdorf MJ. Cartilagenous bone tumors. Radiol Clin North Am 1993:31:237-59.

2. Neben K, Werner M, Bernd L, et al. A man with hereditary exostoses and highgrade non-Hodgkin's lymphoma of the bone. Ann Hematol 2001;80:682-4. 